\title{
Utility and constraints of PocketQubes
}

\author{
J. Bouwmeester ${ }^{1}$ (D) S. Radu ${ }^{1}$ - M. S. Uludag ${ }^{1} \cdot$ N. Chronas $^{1} \cdot$ S. Speretta ${ }^{1}$ - A. Menicucci ${ }^{1}$ - E. K. A. Gill ${ }^{1}$
}

Received: 16 September 2019 / Revised: 20 January 2020 / Accepted: 21 January 2020 / Published online: 5 February 2020

(c) The Author(s) 2020

\begin{abstract}
PocketQubes are a form factor of highly miniaturized satellites with a body of one or more cubic units of $5 \mathrm{~cm}$. In this paper, the characteristics of PocketQubes in terms of their constraints and their (potential) utility are treated. To avoid space debris and limit collision risk, the orbits of PocketQubes need to be constraint. An analysis of orbital decay characteristics has been carried out which, considering existing space regulations and a pro-active attitude, PocketQubes should preferably be launched in low Earth orbits below $400 \mathrm{~km}$ altitude. Due to technical constraints, such as form factor, power and attitude control, the domain of applications for single PocketQube missions is limited. Still, they can act as low-cost training and technology demonstration platforms. To make PocketQubes an attractive platform for other types of missions, not only the launch cost, but also the development, production and operations cost should be significantly lower than CubeSats. When the PocketQube platform matures and produced in high numbers, networks of PocketQubes can enable new applications. Applications considered feasible are in the field of (but not limited to) continuous surveillance using optical instruments, gravity field monitoring using precise orbit determination, in-situ measurements of the space environment, low data rate or bandwidth communication services and inexpensive probes around other celestial bodies.
\end{abstract}

Keywords PocketQube $\cdot$ Application · Orbit · Cost-efficiency $\cdot$ Constellation $\cdot$ Constraints

\section{Introduction}

PocketQubes have been introduced by Prof. Bob Twiggs in 2009 [1]. They are satellites comprising of one or more cubic units of $5 \mathrm{~cm}$ and with a maximum mass of $250 \mathrm{~g}$ per unit. The number of units is typically presented with suffix ' $p$ ' instead of ' $U$ ' as for CubeSats. The original idea was that 8 PocketQubes would fit in a single unit CubeSat (in $2 \times 2 \times 2$ configuration) and could be deployed through existing CubeSat deployers. A cooperation between Morehead State University and GAUSS Srl. has led to the development of a dedicated PocketQube deployment system (MRFOD). Instead of using the corners to slide in the deployment system, like for CubeSats, a baseplate extending a few millimetres from the main body is used which slides in guide rails of the PocketQube deployer. This concept was used for the first ten PocketQubes which have been launched so far, four in 2013 using the MRFOD and six in 2019 using the AlbaPOD,

\section{J. Bouwmeester}

jasper.bouwmeester@tudelft.nl

1 Delft University of Technology, Kluyverweg 1, 2629 HS Delft, The Netherlands and is currently used by the majority of PocketQube developers. Given this deployment system and the additional external envelope, PocketQubes cannot be economically fitted into CubeSat deployers anymore. A publicly available PocketQube standard definition, focussing on mechanical dimensions and interfaces, has been released in 2018 [2].

Besides PocketQubes, several competitive form factors for picosatellites (satellites with a mass between 0.1 and $1.0 \mathrm{~kg}$ ) exist. It is possible to 'slice' a CubeSat into satellites smaller than one unit. For example, Swarm Technologies has launched four SpaceBEE satellites of $0.25 \mathrm{U}$ size into orbit. ThinSats are a competitive standard with a unit size of $11.1 \mathrm{~mm} \times 114.2 \mathrm{~mm} \times 12.5 \mathrm{~mm}$ and a mass per unit of about $280 \mathrm{~g}$ [3]. There are 60 ThinSats launched in April 2019. Both CubeSats slices and ThinSats have a relatively large body surface area compared to PocketQubes, which can be advantageous for solar arrays and 'flat' payloads such as phased array antennas. On the other hand, PocketQubes are more suitable to host payloads which require more depth such as optical instruments. Besides the suitability for specific payloads, the dimensions have an impact on the internal physical architecture, the electrical and mechanical interfaces and attitude disturbance torques. It is, however, not the 


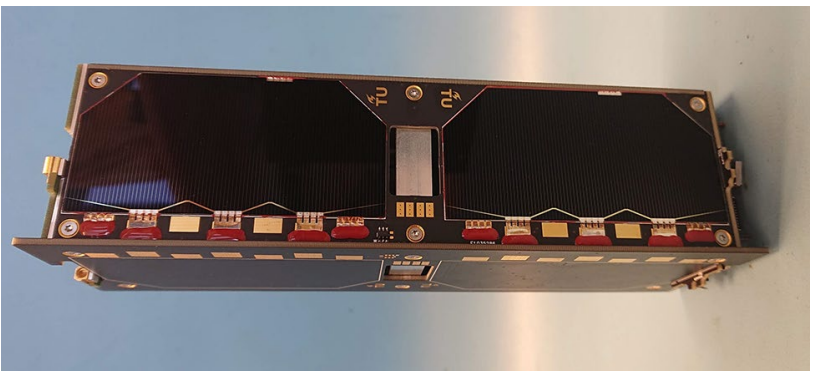

Fig. 1 Photo of partially integrated Delfi-PQ

intention of this study to perform a full trade-off between these competitive standards.

Delft University of Technology is currently developing a 3p PocketQube called Delfi-PQ, which is shown in Fig. 1. A bottoms-up iterative development approach is implemented to improve and demonstrate PocketQube capabilities [4]. Meanwhile, the potential of operational applications with PocketQubes is currently investigated to provide a vision and long term technology development roadmap. This paper provides the first results of this investigation.

At present, the pioneers developing PocketQubes perform research on the limits of satellite miniaturization and use the platform for small satellite technology demonstration. On the long term the sustainability of PocketQubes requires a clear advantage in terms of financial cost effectiveness compared to larger satellites for scientific or commercial applications. In terms of quantity, CubeSats are currently dominant and there is a very good availability of CubeSat components, subsystems or even complete spacecraft busses. For PocketQubes, the investment needed to achieve a similar maturity demands not only a bottoms-up technology development but also on an outlook towards future applications.

This paper provides the boundary conditions for a sustainable future for PocketQubes in terms of legal, regulatory, technical aspects and financial aspects. Also the application domains for PocketQubes are discussed and several examples are provided which look worthy of further investigation. In chapter 2, the conditions for PocketQubes are presented and in chapter 3 potential applications are presented, followed by final conclusions in chapter 4 .

\section{Constraints for PocketQubes}

\subsection{Legal and regulatory guidelines}

Most promising opportunities for applications of PocketQubes are in vast distributed networks. A very important aspect related to this is their risk of contributing to space debris. The Inter-Agency Space Debris Coordination Committee (IADC) stated in 2007: "This IADC and some other studies and a number of existing national guidelines have found 25 years to be a reasonable and appropriate lifetime limit." [5].

The orbital lifetime has been analysed using the free ESADRAMA software tool [6]. First, the effective cross-section in the flight direction has been calculated using the CROC module within the tool. Analysis has been performed on $1 \mathrm{p}$, $2 p$ and $3 p$ satellites with solar panel configurations ranging from solely body mounted up to four-folded solar panel wings. It is expected that deployable solar panels are used on advanced PocketQubes with some form of attitude control for which a uni-directional solar array would be most advantageous. Figures 2 and 3 show the 3D models as used in the CROC module for the most minimalistic and extreme configuration, respectively. The solar panels are $2 \mathrm{~mm}$ smaller (in both dimensions) than the sides of the satellite body. The results are presented in Table 1 .

As can be seen in Table 1, there is a large spread in effective cross section (drag) area depending on the satellite configuration as well as its attitude. The minimum drag area can be achieved with attitude control to extend the orbital life time. Likewise, attitude control can be used to maximize the drag for a de-orbit at the end of the operational lifetime. Since active de-orbit manoeuvres are complex and it can be expected that some PocketQubes may fail prematurely, the best way to assess the orbital life time with respect to space debris mitigation is the non-operational case. Aerodynamic torques can in principle stabilize a satellite with solar panel wings with their small surface in ram direction, but only when there is an energy dissipating actuator to reduce initial momentum and counteract other disturbance torques [7]. Except for satellites which are intentionally designed with a passive attitude control using magnetic hysteresis material,

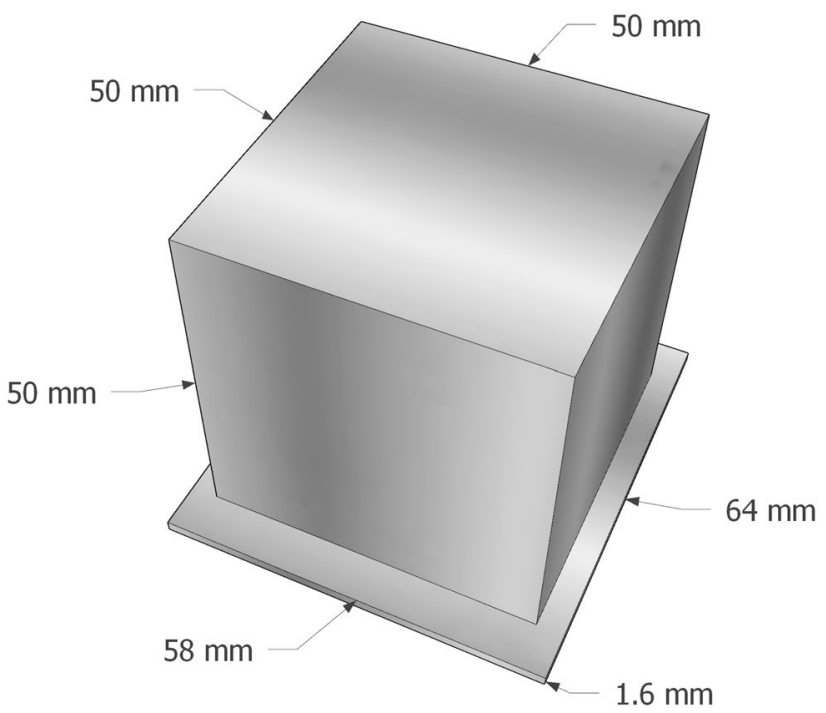

Fig. 2 1p PocketQube with body mounted solar cells 
Fig. 3 3p PocketQube with two quadruple solar panel wings

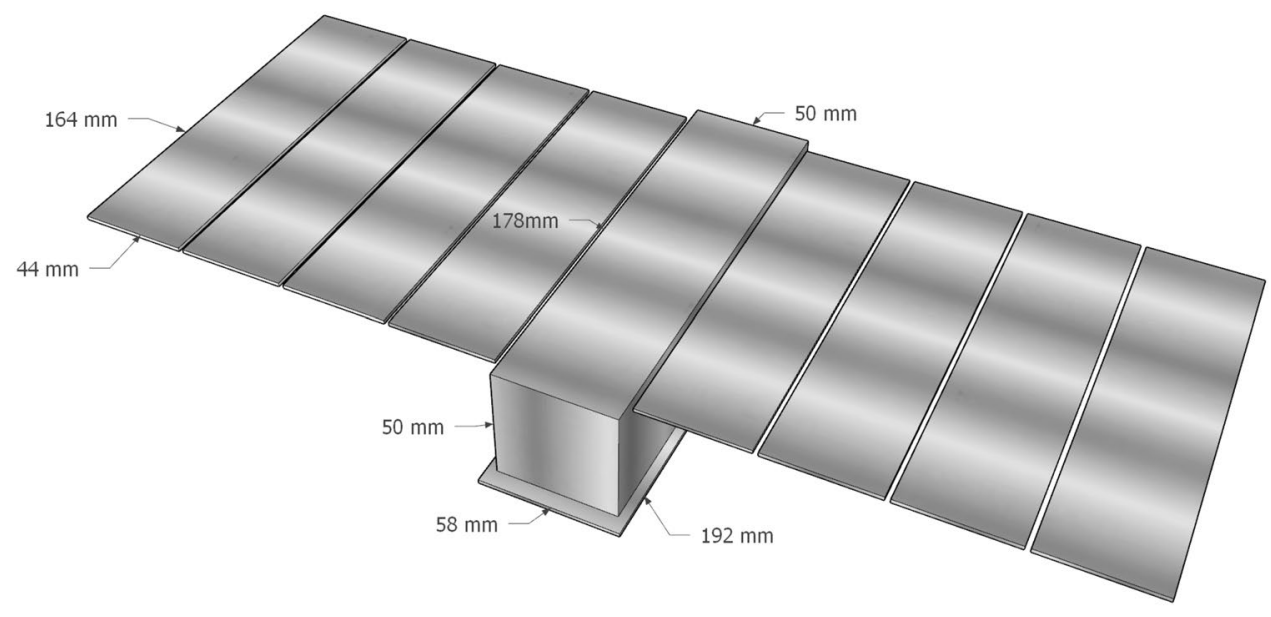

\begin{tabular}{|c|c|c|c|c|c|c|}
\hline \multicolumn{2}{|c|}{ Satellite Configuration } & \multicolumn{3}{|c|}{ Calculated effective cross section area $\left(\mathrm{m}^{2}\right)$} & \multicolumn{2}{|c|}{$\begin{array}{l}\text { Ballistic Parameter } \\
\text { when Tumbling (kg/ } \\
\left.\mathrm{m}^{2}\right)\end{array}$} \\
\hline Unit size & Solar panel configuration & Minimum & Geometric average & Maximum & Minimum & Maximum \\
\hline \multirow[t]{5}{*}{$1 \mathrm{p}$} & Body mounted & 0.0029 & 0.0042 & 0.0048 & 29.8 & 59.6 \\
\hline & Single panel wings & 0.0030 & 0.0057 & 0.0075 & 24.5 & 43.5 \\
\hline & Dual panel wings & 0.0031 & 0.0077 & 0.0112 & 20.4 & 32.5 \\
\hline & Triple panel wings & 0.0031 & 0.0096 & 0.0150 & 18.0 & 26.1 \\
\hline & Quadruple panel wings & 0.0031 & 0.0117 & 0.0189 & 16.2 & 21.4 \\
\hline \multirow[t]{5}{*}{$2 \mathrm{p}$} & Body mounted & 0.0033 & 0.0076 & 0.0092 & 33.0 & 66.1 \\
\hline & Single panel wings & 0.0035 & 0.0108 & 0.0156 & 26.2 & 46.4 \\
\hline & Dual panel wings & 0.0037 & 0.0150 & 0.0244 & 21.0 & 33.4 \\
\hline & Triple panel wings & 0.0039 & 0.0194 & 0.0332 & 17.8 & 25.7 \\
\hline & Quadruple panel wings & 0.0041 & 0.0239 & 0.0420 & 15.8 & 20.9 \\
\hline \multirow[t]{5}{*}{$3 p$} & Body mounted & 0.0038 & 0.0109 & 0.0138 & 34.3 & 68.5 \\
\hline & Single panel wings & 0.0039 & 0.0160 & 0.0244 & 26.4 & 46.8 \\
\hline & Dual panel wings & 0.0041 & 0.0228 & 0.0387 & 20.7 & 32.9 \\
\hline & Triple panel wings & 0.0043 & 0.0300 & 0.0531 & 17.3 & 25.0 \\
\hline & Quadruple panel wings & 0.0045 & 0.0373 & 0.0674 & 15.2 & 20.1 \\
\hline
\end{tabular}

Table 1 Cross section area and ballistic parameter range for different PocketQube configurations it can be assumed that a non-operational satellite is free tumbling. Next to the drag area, the mass of the satellite is an important parameter for the orbital lifetime. The maximum mass per PocketQube unit is $250 \mathrm{~g}$ as defined in the PocketQube Standard [2]. The minimum is assumed to be at least $125 \mathrm{~g}$ per unit for a satellite without deployable panels and an additional $8 \mathrm{~g}$ per unit per panel. This results in a ballistic parameter range for the tumbling scenario, which can be found in the two columns in the right in Table 1. The maximum mass-over-area ratio is found to be $69 \mathrm{~kg} / \mathrm{m}^{2}$ for a $3 p$ PocketQube with body mounted solar cells and a mass of $750 \mathrm{~g}$ in de randomly tumbling scenario. The minimum case is $15 \mathrm{~kg} / \mathrm{m}^{2}$ for a $3 \mathrm{p}$ PocketQube with two quadruple panel wings (see Fig. 3) and a mass of $567 \mathrm{~g}$. With these values, the orbital life time for circular orbits is predicted using the OSCAR module of the ESA-DRAMA tool. A final important input parameter is the launch date since atmospheric densities in low Earth orbit are varying due to the 11-year solar cycle. A sensitivity analysis was carried out which revealed that a launch at the 01-01-2019 provides average results on orbital life-time, while launch dates on 01-01-2024 and 01-01-2028 provides results which are near the lower and upper orbital life time, respectively. Figure 4 provides the results for both the minimum and maximum mass-over-area cases for the three different launch dates.

As can be concluded from the results presented in Fig. 4, a maximum orbital life time of 25 years for PocketQubes can only be guaranteed for circular orbits lower than $630 \mathrm{~km}$ altitude. As the guideline of 25 years has been established before the rapid growth of very small satellite and the 


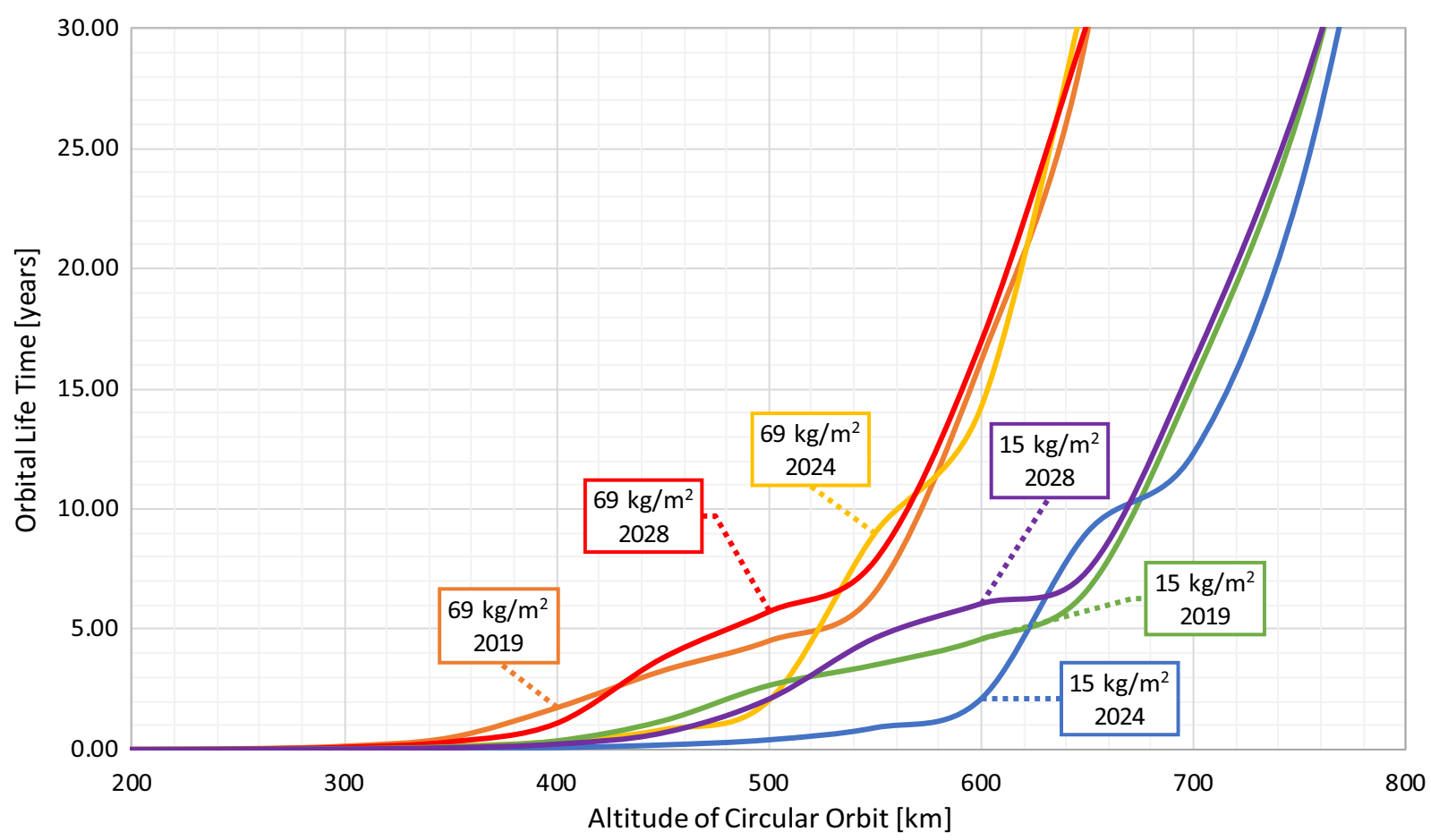

Fig. 4 Orbital life time predictions for PocketQubes in circular orbits

emerging plans for mega-constellations, it can be expected that this guideline will be revised in the near future. For PocketQubes it is recommended to take the more general UN guideline to "Limit the long-term presence of spacecraft and launch vehicle orbital stages in the low-Earth orbit (LEO) region after the end of their mission" [8] in mind and also consider the orbital lifetime in the context of their limited intended operational lifetimes and potentially large infant mortality rate. The authors of this paper, therefore, propose to maximize the orbital lifetime to approximately 5 years regardless of the operational status of the satellite. This implies a maximum of between 480 and $620 \mathrm{~km}$ as can be seen in Fig. 5. Because of this significant spread and the uncertainty of the launch date for some missions, the minimum orbital lifetime during nominal operations should be investigated as well. Furthermore, satellite observability and collision risk are other related aspects which should be taken into account.

Figure 5 provides a zoomed-in graph. In this figure also the orbital life time of the two satellite configurations are presented in case active attitude control is used to minimize the drag over the operational life time. For education and technology demonstration, orbital lifetimes of 3 months to 2 years would be appropriate. For scientific and commercial missions of very small satellites, life times between 1 and 7 years would be appropriate. However, it would be acceptable that active orbit maintenance using on-board propulsion would be required to extend the natural lifetime. Orbits below $300 \mathrm{~km}$, however, seem to be unattractive for PocketQubes as the natural orbital life time will be less than a few months and propulsion with high specific impulse is technically unrealistic for PocketQubes. Because of the low natural orbital life time of Very Low Earth Orbits (VLEO) below $400 \mathrm{~km}$, this orbital regime is not densely populated with space objects yet as shown in Fig. 6. Therefore, the ideal orbital regime for PocketQubes is between 300 and $400 \mathrm{~km}$ altitude for circular orbits. The launch of six PocketQubes on December the $6^{\text {th }}$ of 2019 has been launched to an orbit of $380 \mathrm{~km}$ with an Electron launcher, showing that a launch of PocketQubes to this orbital regime is feasible. Recently, plans for using the VLEO regime for large constellations are emerging such as the request for the Starlink communication network to put 7518 satellites in an orbit of $340 \mathrm{~km}$ to reduce the round trip latency [9]. Also for high resolution imagery VLEO gains interest. This may mean that the ideal orbital regime for PocketQubes will need to be further reduced in the future once these constellations are a reality.

Next to orbital life time, the observability by radar facilities (such as from NORAD) is important as these systems are used to track satellite orbits and calculate collision risks between satellites. Public repositories such as Celestrak provide the tracked orbital parameters by these facilities in the form of Two-Line-Elements (TLEs). A recent study has been performed on the trackability of PocketQubes [10].The four launched PocketQubes are in a near-circular orbit of approximately $600 \mathrm{~km}$ altitude. All four have been tracked 


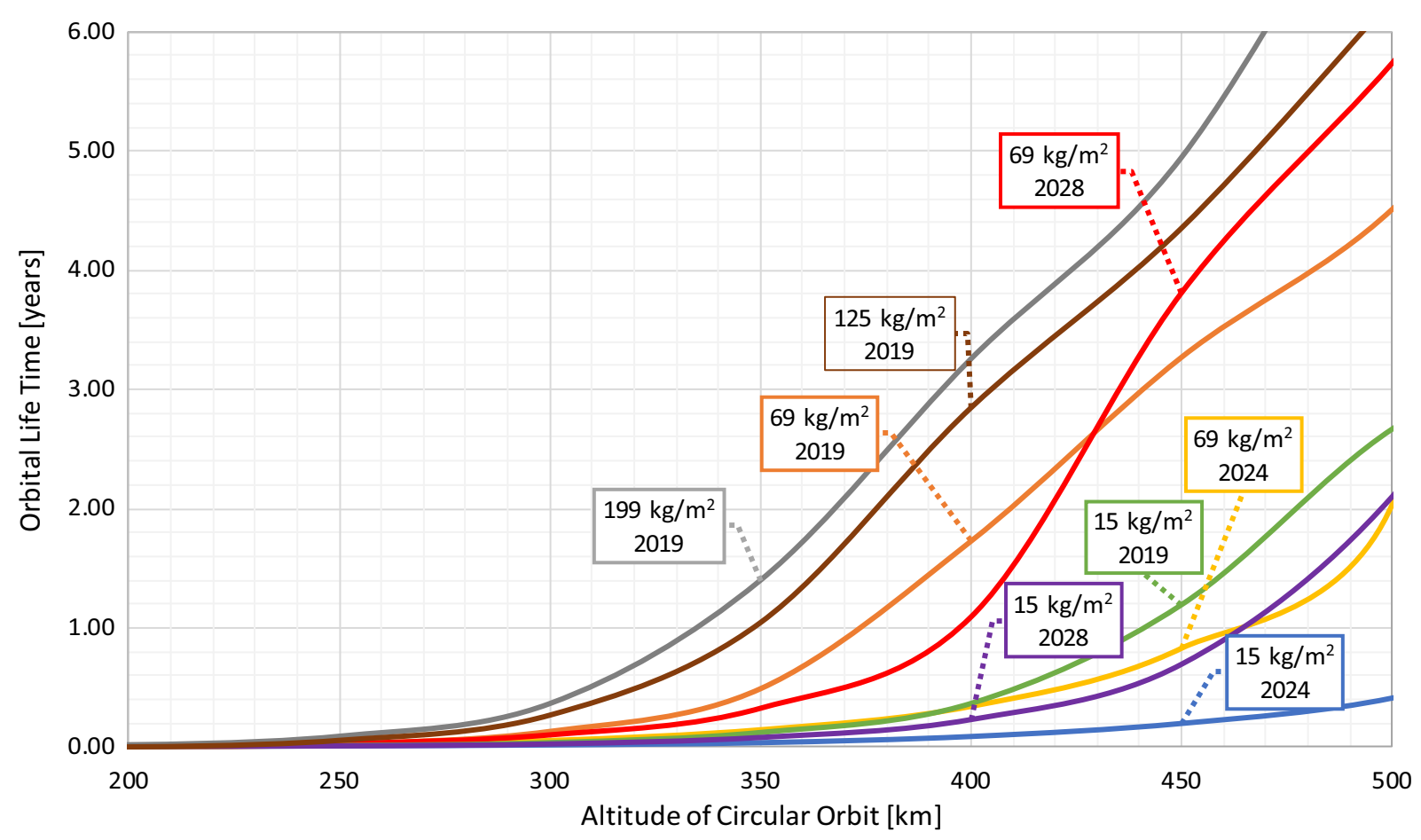

Fig. 5 Orbital life time predictions for PocketQubes in circular orbits (zoomed in)

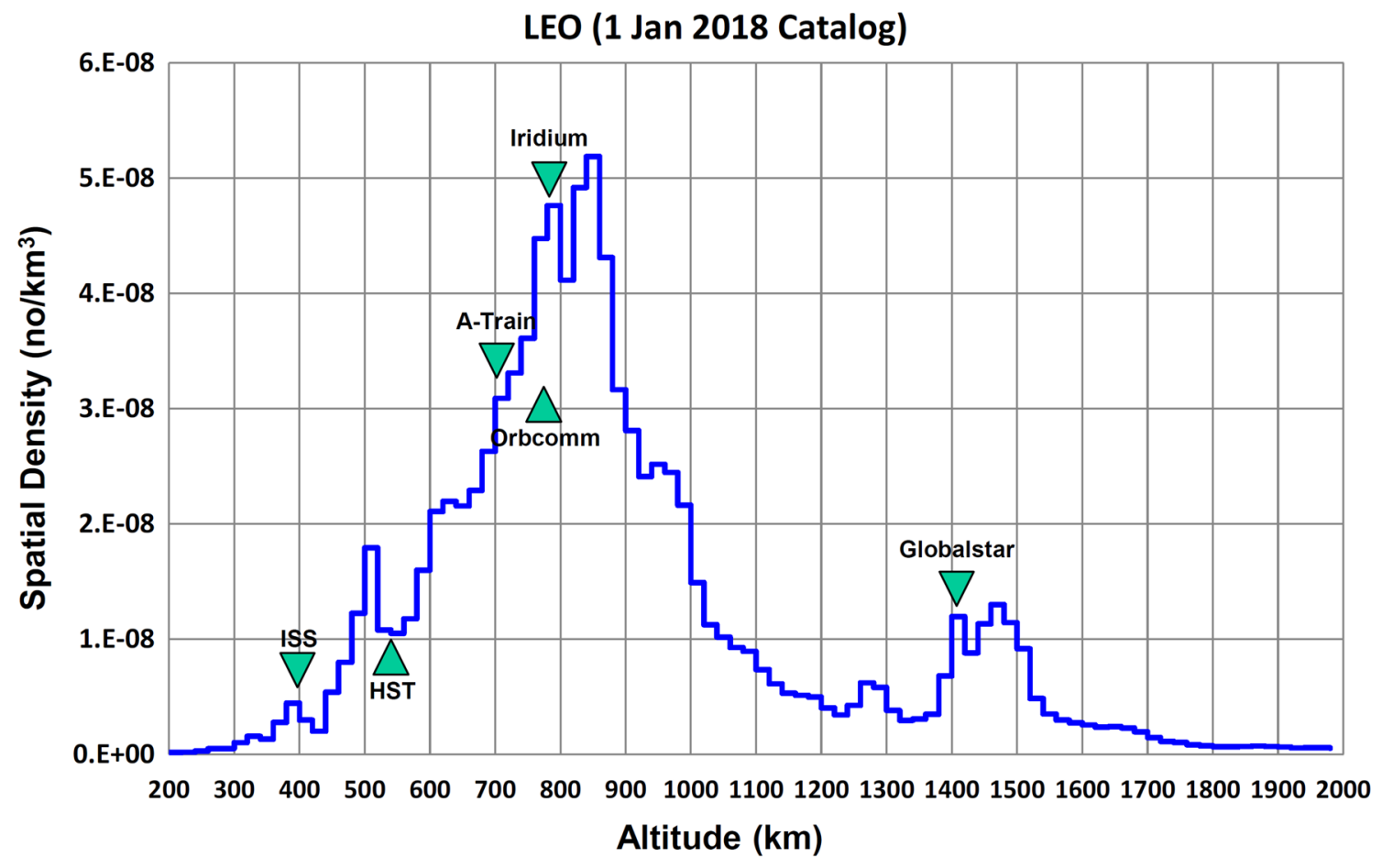

Fig. 6 Density of space objects versus altitude, NASA (CC-PD) [5]

successfully and TLEs have been updated several times per day. However, PocketQubes show a higher covariance between subsequent TLEs compared to larger satellites in similar orbits. Thus, the accuracy of orbit determination with radar detection reduces with spacecraft size. This would also yield a lower accuracy in predicting potential orbital 
collisions. A lower orbit will, however, increase the observability by radar facilities on the ground. The reflected radar signal is linearly proportional to the effective radar crosssection of the object and inversely proportional to the $4^{\text {th }}$ power of the distance (which would be altitude in zenith direction). Although a further study should provide evidence, it is expected that a single unit PocketQube at $400 \mathrm{~km}$ has similar observability to a single unit CubeSat at $600 \mathrm{~km}$. This would again be an argument to launch PocketQubes in the propose orbital regime of 300 to $400 \mathrm{~km}$ altitude.

\subsection{Technical limits and considerations}

The PocketQube is first of all volume- and mass-constrained. Based on the mechanical interface standard [2], the maximum mass per PocketQube unit is $250 \mathrm{~g}$ compared the $1.33 \mathrm{~kg}$ of the CubeSat mechanical interface standard [11], a ratio of approximately five. The volume of a $1 \mathrm{U}$ CubeSat is $1000 \mathrm{~cm}^{3}$ and for a $1 \mathrm{p}$ PocketQube this is $125 \mathrm{~cm}^{3}$, yielding a ratio of eight. However, the allowed thickness of external components and the stand-off distance between single unit satellites outside the main body is not scaled as it is $6.5 \mathrm{~mm}$ for CubeSats [11] and $7.0 \mathrm{~mm}$ for PocketQubes [2] for all sides of the body. The difference of $0.5 \mathrm{~mm}$ is explained by the tolerance taken for CubeSats whereas this is left to the developer for PocketQubes. For multiple unit satellites, the stand-off distance is accumulated in the internal body volume. Taking the external volume as reference, the volume ratio between CubeSats and PocketQubes would be slightly below six. Considering that PocketQube deployers cannot be scaled down linearly in terms of volume and mass compared to CubeSat deployers as well, it can be assumed that one can launch about four times the number of PocketQubes compared to CubeSats in an allocated launch vehicle (slot). This is ratio is a factor two less than the original concept of PocketQubes.

It should be noted that trading PocketQubes against the larger CubeSats will only make sense if the required subsystems and the intended payload do fit in a PocketQube. If the latter is not the case, the PocketQube is simply discarded as an option and only the larger platforms can be considered. This may seem trivial, but it means that one cannot just simple take an existing mission concept for CubeSats (or larger) and trade the utility indicators of a single satellite for a larger number of satellites.

Next to mass and volume, power is another important technical consideration. The orbit average available power for a PocketQube can be just a few hundred milli-Watts for a $1 p$ body-mounted solar panel configurations up to values beyond 10 Watts for a sun-pointed multi-foldable solar array on a 3p PocketQube. Compared to CubeSats, the amount of available power is, based on basic scaling laws, approximately four times less for similar solar panel configurations and the same number of units.

Communication downlink is typically limited by the amount of power. When all other radio link parameters are equal, there is a linear relationship between radio transmission energy and the achievable downlink volume. If the utility of the satellite scales with the amount of data which can be downlinked, this would mean that the utility of a single 1p PocketQube is approximately one fourth of that of $1 \mathrm{U}$ CubeSat. Considering that the same ratio applies to the number of PocketQubes which can be launched compare to the number of CubeSats, the effect of the technical trade-off between the two platforms for networks of satellites would be neutral from an utility perspective, provided that all subsystems and payload can be fitted on both platforms. PocketQubes would allow for a cheaper constellation deployment with respect to CubeSats, leading also to a potentially more congested spectrum. Given that the preferred frequency allocation for small satellites is UHF, additional spectrum has been granted during the World Radio Conference in 2019 [12], providing extra spectrum in the $400 \mathrm{MHz}$ band and also in the $137 \mathrm{MHz}$ band, both dedicated to TT\&C. When attitude control in PocketQubes becomes more advanced, a directional link with higher frequencies can be used for increased bandwidth.

In terms of attitude actuators for such small form-factor platforms, there technical and financial limitations are restrictive and challenging. To be able to perform detumbling and dump momentum build up by disturbance torques, magnetorquers are considered to be the only feasible option. The current attitude determination and control design for Delfi-PQ is shown in Fig. 7 and comprises three custom made magnetorquers and two commercially available integrated inertial measurement units. The system was designed to be able to stabilize the satellite from the

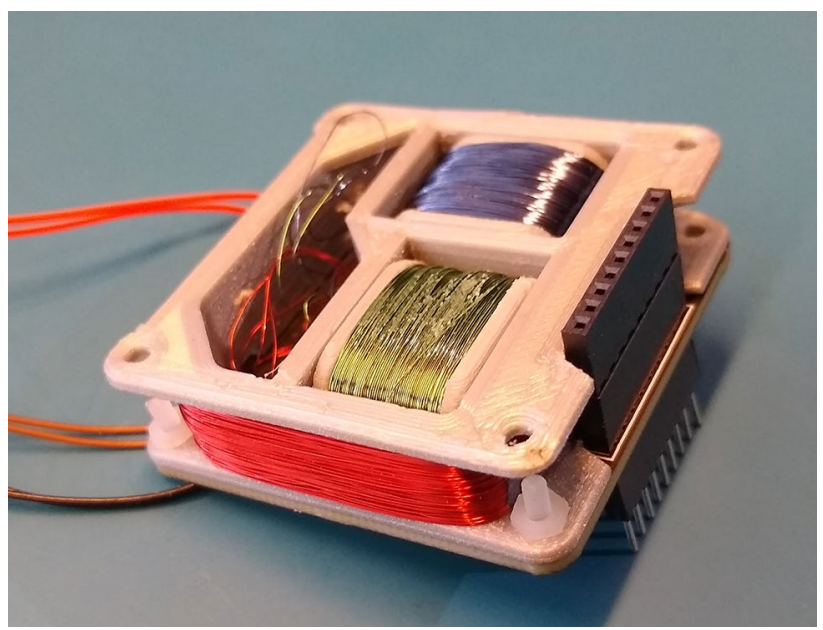

Fig. 7 Delfi-PQ de-tumbling system engineering model 


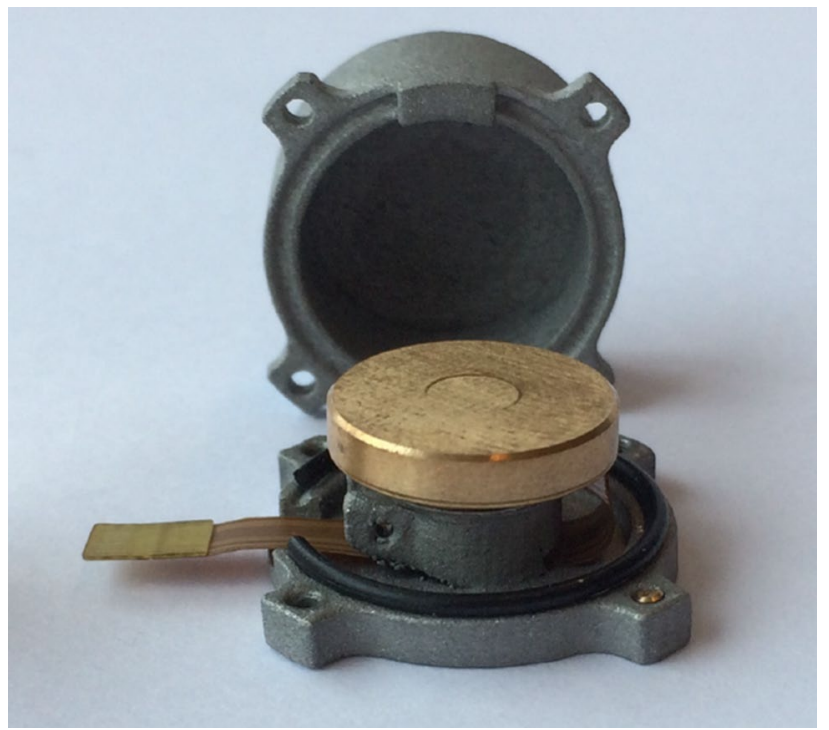

Fig. 8 Prototype of PocketQube reaction wheel

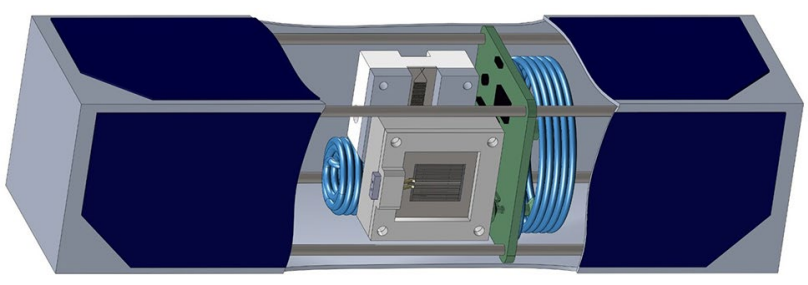

Fig. 9 Artist impression of micro-thruster payload for a $3 p$ PocketQube

maximum rotational speed of $180^{\circ} / \mathrm{s}$ [13]. The de-tumbling stops when the measured rotational speed is $5 \%$ s or lower. For fine control, a reaction wheel for of only $7 \mathrm{~g}$ and steady state power consumption of $25 \mathrm{~mW}$ has been developed at TU Delft [14], as shown in Fig. 8 Prototype of PocketQube reaction wheel. Replacing the lubricant of the bearing with a vacuum proof variant would double the power consumption due to increased friction, which is considered infeasible for the given power budget. A hermetic sealed reaction wheel was developed instead, but the prototype was leaking and further development is pending. Future research should be performed to see if reaction wheels for fine control are truly feasible within the limited technical budgets. A potentially better approach given the limited budgets would be to develop a momentum wheel instead. This would however limit the attitude control to one degree of freedom.

At TU Delft, a versatile micro-thruster is under development which can be adapted either to CubeSats or to PocketQubes by changing the tank size. It is foreseen as payload on Delfi-PQ or its successors and is shown in Fig. 9. As technology demonstration payload, it is equipped with a dual thruster: the Vaporized Liquid Micro-resistojet (VLM) based on vaporizing pressurized liquid water and the Low-Pressure Micro-resistojet (LPM) based on molecular acceleration of propellant molecules stored at low pressure [15]. Moreover, the micro-propulsion system consists also of the tank, the feed system and the afferent electronics board. The main goal of the micro-propulsion payload is to demonstrate, test and compare different resistojet technologies. The current specification is a thrust between 0.1 and $3 \mathrm{mN}$ with a specific impulse from 50 to $100 \mathrm{~s}$. The overall estimated mass of this system, including propellant is around $75 \mathrm{~g}$ and the peak power consumption of the system during trust is below $4 \mathrm{~W}$.

Active thermal control on PocketQubes is very challenging due to the limited available power. With passive thermal control it is possible to limit the cold and hot temperatures for typical operational ranges for electronic components. Active cooling for instruments is very difficult to achieve with PocketQubes.

Potential payloads are constrained by the volume of the PocketQube. This poses physical boundaries on what can be measured, for instance on the signal-to-noise ratio and the resolution. For optical instruments, the diffraction limit is provided by Eq. 1 .

$\theta_{\min }=a \sin \left(1.22 \frac{\lambda}{D}\right)$

where $\theta_{\min }$ is the diffraction limited angular resolution. $\lambda$ is the wavelength. $D$ is the diameter of the entrance pupil (aperture) of the imaging lens.

The aperture for a PocketQube camera would practically be limited to approximately $4 \mathrm{~cm}$. However, the limited length of the optics inside the camera can also be a driving factor which may lead to an even smaller achievable aperture. In Fig. 10, the diffraction limited Ground Sampling Distance (GSD) is provided for a $2 \mathrm{~cm}$ and $4 \mathrm{~cm}$ aperture camera for at $300 \mathrm{~km}$ and $400 \mathrm{~km}$ altitude looking in zenith direction. The achievable GSD is approximately half of what can be achieved with a linearly scaled CubeSat camera at the same orbit.

Given a maximum total launch volume and/or mass, the advantage of the PocketQubes could be that it provides more simultaneous measurements (for in-situ monitoring). As long as all payload data can be downlinked by each PocketQube, there will not be any penalty. If this is not the case, the utility of the mission is a trade between the number of simultaneous measurements (satellites) versus the amount or rate of sequential measurements which can be downlinked. Likewise, temporal resolution of Earth observation of the same areas on Earth can be increased with PocketQubes compared to CubeSats, potentially at cost of the amount of coverage due to reduced downlink volume per satellite. For single satellite PocketQube missions there is no technical advantage over CubeSats. They can, at best, be equal 


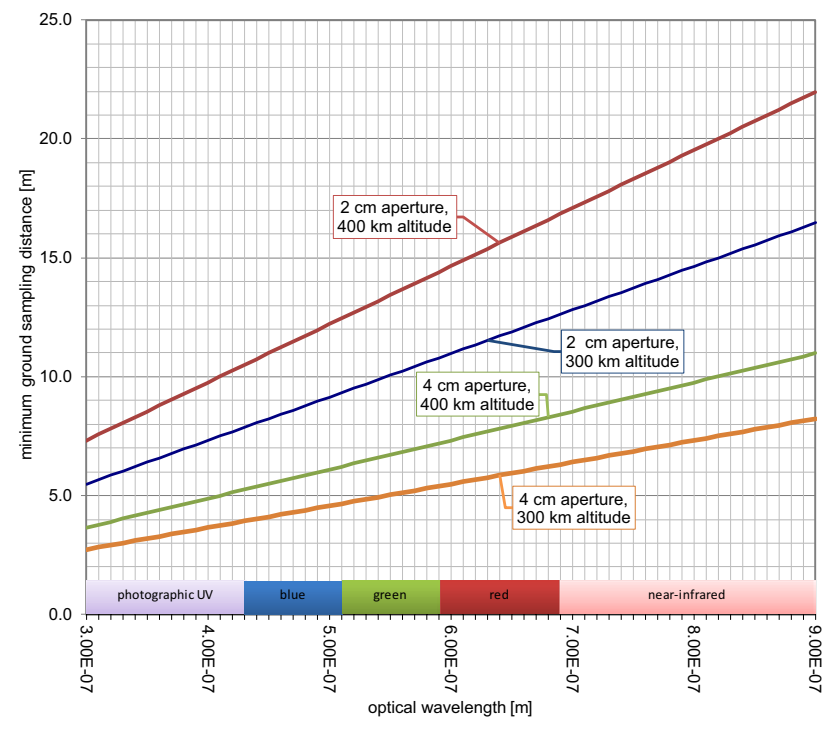

Fig. 10 Ground sampling distance of diffraction limited cameras

if the utility of the satellite is not limited by the size of the PocketQube in any way (mass, volume, data downlink, duty cycle, etc.).

\subsection{Financial conditions}

According to a study in 2014 on small launch vehicles, the specific launch cost to LEO ranges from $7 \mathrm{kUSD} / \mathrm{kg}(\sim 5 \mathrm{k} € /$ $\mathrm{kg})$ for a PSLV-CA launch to $45 \mathrm{kUSD} / \mathrm{kg}(\sim 33 \mathrm{k} € / \mathrm{kg})$ for a Pegasus XL launch [16]. These are gross prices based on the total mass capacity. For PocketQubes and CubeSats, the actual price will also be based on the mass of the deployer, the administrative overhead at the launch provider and the services provided by the any intermediary parties (launch brokers/service providers). According to the same study, the launch of a $3 \mathrm{U}$ CubeSat in a P-POD on an Athena-IIc launch vehicle is $300 \mathrm{kUSD}$ fir a $5 \mathrm{~kg} 3 \mathrm{U}$ CubeSat, yielding a net satellite specific launch cost of $60 \mathrm{kUSD} / \mathrm{kg}$, while the gross specific launch cost for this vehicle is specified to be 20 $\mathrm{kUSD} / \mathrm{kg}$ [16]. Emerging very small launch vehicles, such as the Vector-R and the Electron are estimated (based on information in public announcements) to provide launch at gross specific cost of $25 \mathrm{kUSD} / \mathrm{kg}$. This is significantly higher than for the medium class PSLV. However, since these vehicles can launch CubeSats and/or PocketQubes only, it is expected that the administration, handling, safety procedures and services can be tailored and optimized such that the net specific cost per satellite will approach similar figures as for larger launch vehicles. At the PocketQube workshop held in 2018 in Delft, the prices presented for PocketQube launches were $20 \mathrm{k} €$ per unit. With a maximum mass of $250 \mathrm{~g}$ per unit, this yields a net specific launch cost of $80 \mathrm{k} € / \mathrm{kg}(\sim 100 \mathrm{kUSD} /$ $\mathrm{kg}$ as of April 2018). This is slightly higher than the specific launch cost for CubeSats, which can be explained by the fact that administrative overhead and services do not scale down linearly with satellite mass. There are several ways to achieve a lower launch cost per PocketQube unit:

- Optimizing the mass of a (containerized) deployment system with respect to the satellite mass. This could potentially be achieved by batch deployment systems (e.g. a 96p deployer as proposed by Alba Orbital Ltd.).

- Standardizing and automating launch procurement, administration and pre-launch procedures to reduce overhead and service cost.

- Increased competition in the field of (small) launch vehicles, driving down the gross specific launch cost.

Depending on the success and implementation on the developments described above, it is expected that the net specific cost for PocketQubes of $40 \mathrm{k} € / \mathrm{kg}(50 \mathrm{kUSD} / \mathrm{kg})$ should be feasible in the future. This would yield $10 \mathrm{k} €(13$ kUSD) per PocketQube unit. The ideal ratio of launch cost between CubeSats and PocketQubes would be a factor four based on the effective volume and mass ratio provided in Sect. 2.2.

The launch cost is part of the overall mission cost. The potential utility of PocketQubes per satellite is smaller than that of CubeSats as explained in the previous section. When PocketQubes will grow beyond their infancy and reach a maturity level comparable to CubeSats, satellite developers will be required to select the appropriate size of satellite to maximize the cost in relation to the utility. A paper on 'rightsizing small satellites' has investigated this [17]. According to D. Barnhart: "A concerning trend in small satellite industry is our fixation on a particular small satellite standard, namely the $3 \mathrm{U}$ CubeSat. While the $3 \mathrm{U}$ CubeSat is an excellent choice when the payload can readily fit without modification, the mission cost can skyrocket when the payload is purposefully miniaturized to fit $3 \mathrm{U}$ CubeSat. The primary author has personally witnessed several payload development programs with this aim. The result, in every case, was program failure. These efforts to force-fit high functioning payloads within a $1.5 \mathrm{U}$ payload space proved to be costprohibitive" [17]. These are lessons learnt which should also be taken into account when PocketQubes are considered.

When considering mission applications for PocketQubes, a simple guideline would be that the total of the development, production and operations cost per satellite should ideally be on par with the launch cost. The rationale behind this is that if the development, production and operations cost would grossly exceed the launch cost, it would be appropriate to consider larger spacecraft (such as CubeSats) to increase the utility per satellite to optimize the overall utility per total cost. Likewise, if the launch cost would very dominant, it would make sense to investigate if further 
miniaturization would be physically feasible and be more cost-effective. Development cost is mainly based on human resources and the difference between a sophisticated CubeSat or PocketQube development may not be very different when looking at effort. The only way to reduce the development cost per satellite is by producing and launching many identical satellites such that development cost can become a minor contributor to the overall cost. If for example the development cost of a sophisticated small based mission is $10 \mathrm{M} €$, the development cost per satellite is still $100 \mathrm{k} €$ per satellite for network a 100 satellites which could be justified for CubeSats, but at 1000 satellites it becomes $10 \mathrm{k} €$ for which PocketQubes could be financially attractive. Also production cost (including unit testing, assembly and integration) should be significantly lower than for CubeSats. This will not be achieved by the reduction of materials only. Smart architectural concepts such as the integration of several subsystems and a quick assembly of integrated outer panels [18] as well as automated unit testing can be ways to make a larger differentiation in terms of cost per satellite.

\section{Potential applications for PocketQubes}

Taking the conditions discussed before into account, this chapter provides some insight into potential applications of PocketQubes. This is not meant as an exhaustive analysis or a complete overview, but to show the potential of PocketQube platforms and provide some of the main considerations to this respect.

\subsection{Training and education}

Education and training on real satellite platforms is more inspiring and provides a deeper learning experience than only theory or exercises. While each class of satellites has differences in development approach and technology, the basics of spacecraft technology are platform independent and it is always possible to apply the more extensive approaches of larger spacecraft on the smaller platform for training and education purposes. Therefore, the cost-effectiveness of a PocketQube platform is a major advantage for training and education. While CubeSats have opened space to many new players around the world, it is still cost-prohibitive for many small companies and a majority of educational institutions. PocketQubes have the potential to open the doors for an even wider range of players. For PocketQubes the technology is not yet as mature as for CubeSats and for many (advanced) subsystems. There are not many modules on the market while for CubeSats the commercial available subsystems are advanced and diverse, which poses a dilemma for academic teams as educational/training objectives are sometimes in conflict with the desire to keep up with the state-of-the-art which typically would require procurement of commercial subsystems. For PocketQubes there is still a need for development of this technology starting from a low technology readiness level and basic performance, which at present provides excellent opportunities to align research, development and education objectives at universities.

To this respect, the experience of building a PocketQube platform in a strongly education-oriented context represents an ideal continuation of the previously taken steps within the Delfi Program. The Delfi Program is a frame under which satellites are built by TU Delft since 2004 in a collaborative effort by staff, students and industrial parties to contribute to miniaturization of space systems and demonstrate innovative space technology. The program has three main objectives: education, technology demonstration and platform innovation. The program supported many MSc theses and students projects at various levels. More than 150 students have participated in the program so far, and many of them have found a career in space. Delfi- $\mathrm{C}^{3}$ was the first CubeSat launched by a team on April $28^{\text {th }}$ of 2008 in the frame of the Delfi program, and it is still operational. The satellite was developed with the technology, facilities and support of project partners (Airbus DS, TNO, NLR, etc.) which enhanced the visibility of the students within space industry and their future opportunities. Delfi- $\mathrm{C}^{3}$ was followed up by Delfi-n3Xt which was launched on 21st of November 2013 and completed its primary mission goals within 3 months after which it became non-operational. A spin-off company Innovative Solutions In Space (ISIS) was established in 2006 based on the experience gained through the contributions to the design of Delfi- $\mathrm{C}^{3}$. It is currently one of the world leaders in the small satellite market.

The Delfi program currently continues with Delfi-PQ, the first PocketQube in line. With the lower cost, TU Delft wants to promote the idea of always having one or more space systems at hand in the cleanroom. This enables students to gain access to design and development of real space-systems and understand through their work what the end-to-end development of a unit/subsystem/satellite represents.

\subsection{Technology demonstration}

PocketQubes are excellent platforms for low cost technology demonstration, provided that the demonstrated object or concept fits in a PocketQube platform. Demonstration of technology can be that of PocketQube-sized subsystems or components itself, but it can also be performed for technology which is meant for larger platforms. For instance, sun sensors of a large spacecraft are typically still small enough to fit in a PocketQube. If this would be the only demonstration payload, the technical requirements would be relatively modest. A technical challenge however may be that for perfect in-orbit demonstration or even characterization, the 
PocketQube should be able to provide additional high quality and reliable reference measurements and the platform should, therefore, be mature. In the case of the Sun Sensor example, the attitude determination of the PocketQube should provide a good independent attitude reference.

PocketQubes could also be good platforms to demonstrate novel concepts and algorithms which are in principle platform-size independent. Concepts can be based on new architectural approaches [18], novel communication techniques but also on distributed systems such as formation flying and rendezvous and docking. Novel attitude algorithms can be tested on PocketQubes, provided that it fits within the available computational power and does not require large attitude sensors.

\subsection{Earth observation}

Earth imagery can be performed with very small satellites, such as the 3U CubeSats of Planet using a platform optimized diffraction limited camera. Planet has the ambition to monitor the Earth daily at a ground sampling resolution of 3-5 m with a network of about 200 satellites [19]. For PocketQubes, the diffraction limit will limit the achievable Ground Sampling Distance (GSD) to half of that of a CubeSat of similar configuration (see Sect. 2.2). As the data produced per satellite has a quadratic relationship with GSD, just like the required power to downlink the data, the amount of imaged land area per satellite per time unit can be identical between PocketQubes and CubeSats. As there are already many CubeSat developers active in the field of Earth imagery and plans are being developed for large constellation for Earth observation, using PocketQubes only makes sense for those applications which can still provide a complimentary function. Performing the same type of mission as Planet at, for example, half the GSD and four times the temporal resolution may not be the right approach since starting from scratch will make it difficult to be cost-competitive against a scale-up of the existing solutions. Potential applications in Earth imaging can better be found in niche applications requiring large constellations which cannot be easily performed by Earth observation constellations with general utility aimed at mass markets. An example of a potentially useful application would be to monitor cloudy regions, such as the Netherlands, from different angles to maximize the change that images of the ground can be shot. Another example would require simultaneous multipoint measurement with full Earth coverage. Earth radiation, the combination of the reflection of Sunlight (Albedo) and its own body radiation (infrared dominant), provides valuable information for climate models. In a study on CubeSats for Earth observation it has been found that uncooled microbolometers are suitable for CubeSats [20]. As these instruments are not diffraction limited, they require little power without the need for active cooling and provide relatively low data volumes if they measure only one ground sample at a time, they could also be suitable for PocketQubes. With a well distributed constellation of small satellites, it would be possible to monitor the whole Earth continuously and even provide insight into regional differences.

For Earth observation in general, it may pay off to investigate if commercial available cameras or integrated imaging sensors for terrestrial applications can be used as-is or with minor modifications for specific purposes. If successful, this may lead to cost-effective solutions which are very different from how CubeSat instruments are currently being developed and implemented.

Global Navigation Satellite System (GNSS) receivers which have two or more frequencies or feature real-time kinematics can be fitted inside a PocketQube [24]: multiple receivers from several suppliers, developed for the consumer market, are available with a footprint of less than $2 \mathrm{~cm}^{2}$ and a total consumption of less than $0.5 \mathrm{~W}$. Typical accuracies are in the order of few centimetres: they could be used for measuring the gravity field of Earth such as performed for GOCE, CHAMP and GRACE [25] but with a more limited accuracy. According to the study on Earth observation for CubeSats, this was deemed infeasible "as it requires extremely fine attitude control and a very low orbit that would necessitate a continuously operating propulsion system" [20]. The advantage of PocketQubes, however, is that it approaches the ideal point-mass for these types of measurements and as such the requirements on the attitude can be relaxed to a few degrees to ensure that the receiver can lock onto the signals and the physical measurement offset is limited to less than a millimetre. For the L1 GPS frequency at $1575 \mathrm{MHz}$, an effective quarter-wavelength patch antenna would $48 \mathrm{~mm}$ wide, which is exactly possible on a PocketQube. A maximum determination offset of $1 \mathrm{~mm}$ would yield a maximum phase offset of just 0.03 rad and would be excellent for precise orbit determination. As discussed in Sect. 2.1, the ideal orbital regime for PocketQubes is actually in line with the required very low orbits for GNSS based gravity field measurements. It is affordable to dispose the PocketQubes after a limited lifetime and replace them regularly.

\subsection{Space weather observation}

The foreseen low orbit regime of PocketQubes limits the scope of applications of space weather, but the dynamics of the Earth magnetic field in combination with the radiation penetrating into the thermosphere can still cover a gap in science when simultaneous multipoint measurements are required. Also the effect of radiation in LEO on electronics can be investigated with statistical relevance using a network of similar PocketQubes. 
Science grade magnetometers can fit in a PocketQube as physics allow them to be small and the data rates are not extremely high. For example, a scientific fluxgate magnetometer which was developed for CubeSats with sensor dimensions of $36 \mathrm{~mm} \times 32 \mathrm{~mm} \times 28 \mathrm{~mm}$ and a power consumption of $400 \mathrm{~mW}$ [21] would in principle also fit in a PocketQube. The influence of the other electronics in the satellite needs to be mitigated, for example with boom similar as the one developed for the CubeSat payload [21]. At TU Delft, an ongoing study investigates if the need for a boom can be omitted by distributing several magnetometers within the spacecraft to be able to differentiate between the external magnetic field and local disturbances. As the geomagnetic field is very dynamic, "Multiple satellite missions measuring simultaneously over different regions of the Earth offer the only way to take full advantage of the enormous improvement in instrumentation that has been achieved during the last years." [22]. A constellation of PocketQubes can push the limits of in-orbit spatial resolution in a cost-effective way.

Very small radiation sensors, such as the Highly Miniaturized Radiation Monitor (HMRM) of only $1.7 \mathrm{~cm} \times 2.4 \mathrm{~cm} \times 2.2 \mathrm{~cm}$ [21], can be fitted inside a PocketQube. However, the power consumption of these sensors remains too high for the PocketQube platform. To meet the power requirement, other novel methods to detect particle radiation are currently studied at TU Delft, such as the 3D NAND memory based sensors which aim to detect single events particle tracks as well as other type of commercially available electronic components (e.g. Floating Gate Dosimeters) for monitoring total ionization dose in a cost effective and relatively easy-to-implement manner. Although the radiation environment in LEO is generally benign, the potential scientific application for PocketQubes based particle radiation instruments in these orbits is best exploited by a distributed network of sensors ideally on-board of a constellation of satellites. The analysis of the measurements provided by the radiation sensors can bring valuable insights on the dynamicity of space weather in time and position.

\subsection{Communication services}

Communication as a service for PocketQubes is extremely limited by the available electrical power. The data volumes and/or signal bandwidth which can be achieved is prohibitive for commercial services base on mass market telephony or internet. However, it is still possible to use PocketQubes for services which require low data volume downlinks and/ or bandwidth. The areas of interest are spectrum monitoring, Internet-of-Things (IoT) and individual communication services. Spectrum monitoring using software defined radios can be used to identify the global, regional and local use of the radio frequency spectrum. PocketQubes are not suited for missions targeting broad spectrums, for example a the HawkEye 360 pathfinder missions [26], which require more complex antennas (and thus bigger). Identification of underutilized bands, discovery of perpetrators (radio pirates) or defence related intelligence (e.g. use of radars, jammers, etc.) are very difficult to achieve in PocketQubes due to the low available power for on-board computations and limited downlink capability. PocketQubes are more suited for smaller mission, targeted at specific signals, like monitoring the vertical leakage from TV towers as being demonstrated on the Smog-1 PocketQube [27]. The small form factor and low cost make them ideal for a dedicated mission, rather than at a generic system, benefitting from a fast development cycle and potentially frequent launches.

With the advance of IoT devices on Earth, in-situ monitoring vast amounts of areas and objects of interest becomes possible provided that the signals of these monitoring devices can be picked up. For remote locations, such as the arctic [28], a satellite constellation may be more cost-effective than installing ground based reception systems. The data rates produced by the ground-based sensors are very limited, typically in short messages of a few bytes sent only once per few minutes to hours. With an increasing number of sensors, it becomes interesting to perform a trade-off between several classes of satellites to optimize for the total data bandwidth but also to properly deal with mutual interference.

The final application related to commercial services could be to allocate a single PocketQube to one or only a few telephony or low bandwidth data communication channels. This should in principle be feasible with a PocketQube. While a constellation can improve the continuous availability and linearly scale up the number of available channels, it will not be feasible to address the bulk consumer market. The applications would, therefore, be limited to military and emergency purposes. As they are likely not competitive to (existing and emerging) networks of larger satellites, the potential advantage can be found in the fact that a dense network of PocketQubes would be very difficult to taken out of operation. Also in case of a major disaster, for example related to an extreme solar particle event, PocketQubes may be used to quickly set up a global communication network for critical (but limited) communication services.

\subsection{Space exploration}

PocketQubes could potentially go beyond Earth orbit, e.g. to the Moon or other planets in our solar system, using rideshare capacity of larger satellite missions. The main advantage is this case is that the cost per mass or volume unit to these orbits is significantly higher compared to LEO, which would be a stronger motivation for further miniaturization. This could justify a higher development cost per satellite following the argumentation in Sect. 2.3. 
Because of the electrical power limitations and the large communication distance, a large 'mother-satellite' acting as relay would most likely be required in the near to medium term future. Applications could be in the field of distributed networks of PocketQubes to increase temporal resolution or for spatially distributed in-situ measurements around other celestial bodies. They could create a constellation to establish a navigation system at that specific body (similar to GPS) and perform space weather monitoring (for space situational awareness). The PocketQubes could also be dropped on the respective surface of celestial bodies as probes. A concrete example of this potential application can be related to a specific Lunar mission in which Delft University of Technology is participating, called LUMIO [29]. LUMIO is a $12 \mathrm{U}$ CubeSat that will orbit around the Earth-Moon Lagrangian point L2 to monitor the micro-meteoroid impacts that occur on the Lunar far side, which can be identified and characterized through the flashes they make at impact. The optical observations performed by LUMIO could be potentially combined, to cross-check and validate their outcomes, the measurements performed by in-situ PocketQubes that are distributed and dropped on the far-side of the moon and can act as seismographs.

There are major technical challenges in the field of radiation tolerance and thermal control. Most PocketQube technology at present is developed with commercial of the shelf electronics for terrestrial purposes, which is unsuitable as-is for the harsh radiation environment and high ionization doses encountered outside the LEO environment. PocketQubes have a relatively high ratio of outer surface area over internal heat capacity compared to larger satellites, making thermal control outside the Earth's orbit around the Sun very challenging. The very limited available electrical power makes it difficult to compensate this with active heating or cooling. Studies on using CubeSats on interplanetary missions provide a roadmap for these class of satellites beyond LEO [30]. In this study, an $8 \mathrm{~mm}$ thick Aluminium shielding is proposed to tackle the radiation issue. While it is questionable if such shielding provides adequate protection against all types of particle radiation effects, such shielding would in any case be prohibitive for PocketQubes. The question whether PocketQubes can provide useful and cost-effective platforms beyond LEO requires a dedicated study with a critical comparison with larger platforms at a mission level, taking the utility and overall cost into account. Finally, they can be a step towards further miniaturization of satellites which can eventually, in combination with laser/ solar sails or with multistage electric propulsion, push the boundaries of satellite distance. An exotic mission such as Breakthrough Starshot, where tiny spacecraft will be sent to Alpha Centauri [31], creates an ultimate frontier to work towards and PocketQubes can be used to demonstrate the first steps.

\subsection{Overview of applications}

In Table 2, an overview is provided to summarize the described applications and complimented with the foreseen feasibility and required maturity for implementation.

\section{Conclusions}

PocketQubes are a new class of very small satellites which can enable new types of applications provided that technical, legal, regulatory and financial constraints are properly taken into consideration. Single satellite PocketQube missions are ideal for education and training purposes as well as a limited set of technology demonstration objectives.

For Earth observation and communication services, the will most likely only show their true potential in vast distributed networks of hundreds to thousands of satellites. With these numbers, a conservative approach should be taken on the risk to create space debris. The orbit of PocketQubes should be below $500 \mathrm{~km}$ of (average) altitude to limit the orbital life time to 5 years, with a preference to limit it further in the future to circular orbits between 300 and $400 \mathrm{~km}$ while using the potential of on-board propulsion to extend the operational lifetime.

For a sustainable PocketQube mission, the launch cost should be approximately four times less than that of a CubeSat and the total of development, production and operations cost per satellite should ideally be on par with the launch cost. The volume and mass constraints of a PocketQube lead to less available electrical power compared to CubeSats and also physically limits the achievable measurements or services. Instead of simply scaling down the instruments of CubeSats and increasing the number of satellites, it is advocated that further differentiation from existing and planned CubeSat missions may be more promising. For Earth observation, continuous monitoring of dedicated areas of Earth or globally distributed simultaneous measurements of particle radiation, the Earth magnetic field or the Earth infrared radiation would be vastly different from the concepts of today where the Earth is monitored on a daily basis. For communication services, low data rate and/or bandwidth applications as well as specific RF spectrum monitoring could enable niche applications, but further investigation is required to compare those concepts with respect to larger satellites in lower numbers. PocketQube missions beyond Earth may have their potential as probes or used in a constellation and could be cost competitive to larger platforms due to increased launch cost per mass unit, but challenges such as the harsh radiation environment and thermal challenges will require a major advancement of the platforms.

Overall, it can be concluded that PocketQubes provide sufficient potential for present and future applications. 


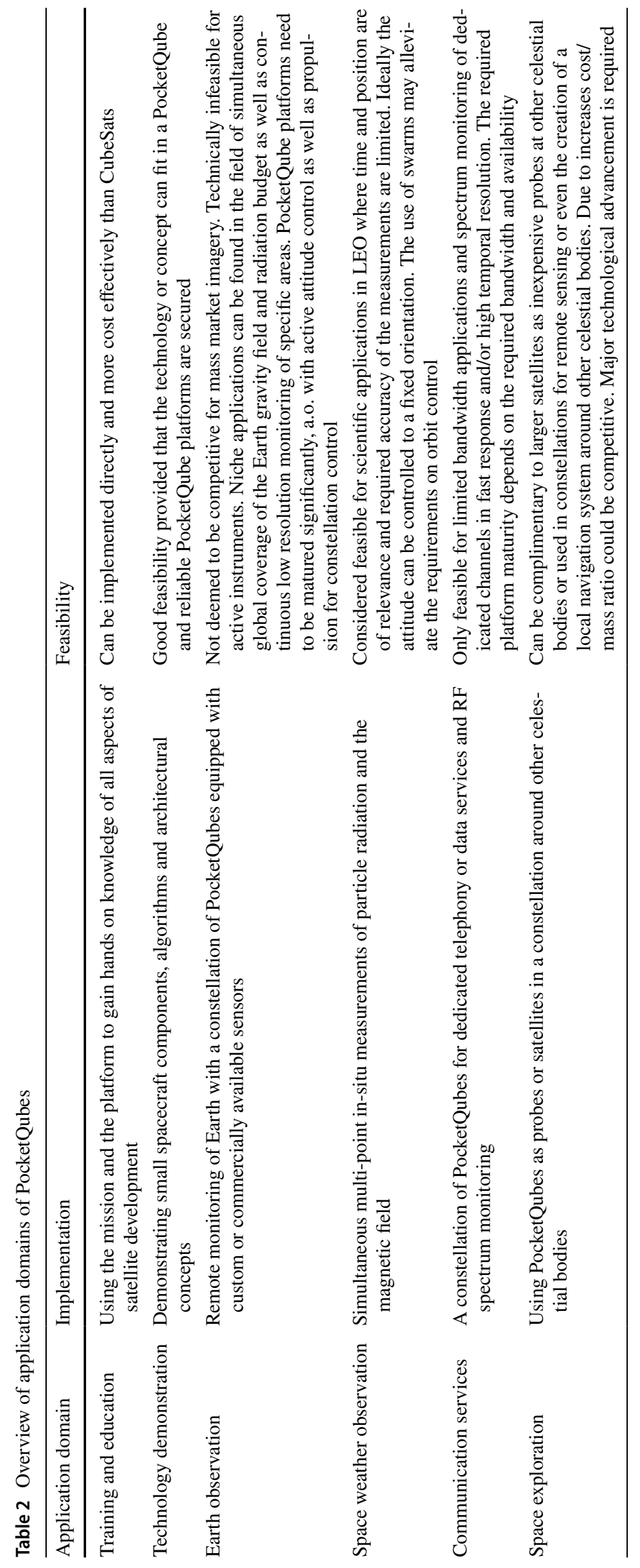


Instead of being competitive platforms to CubeSats, they should be regarded as a complementary class of satellites which enable new cost-effective applications.

Open Access This article is licensed under a Creative Commons Attribution 4.0 International License, which permits use, sharing, adaptation, distribution and reproduction in any medium or format, as long as you give appropriate credit to the original author(s) and the source, provide a link to the Creative Commons licence, and indicate if changes were made. The images or other third party material in this article are included in the article's Creative Commons licence, unless indicated otherwise in a credit line to the material. If material is not included in the article's Creative Commons licence and your intended use is not permitted by statutory regulation or exceeds the permitted use, you will need to obtain permission directly from the copyright holder. To view a copy of this licence, visit http://creativecommons.org/licenses/by/4.0/.

\section{References}

1. Twiggs, R.: Making it Small. Cal Poly Developers' Workshop, San Luis Obispo (2009)

2. Radu, S., et al.: The PocketQube standard (2018)

3. Twiggs, R., et al.: The ThinSat program: flight opportunities for education, research and industry. In: 32nd Annual AIAA/USU Conference on Small Satellites (2018)

4. Speretta, S., et al.: CubeSats to Pocketqubes: opportunities and challenges. In: Proceedings of the International Astronautical Congress (2016)

5. Inter-Agency Space Debris Coordination Committee. IADC space debris mitigation guidelines. IADC Sp. Debris Mitig. Guidel., Revision 1, pp. 1-10 (2007)

6. Gelhaus, J., et al.: Upgrade of DRAMA, ESA's space debris mitigation analysis tool suite. In: 6th European Conference on Space Debris, vol. 2013, no. April, pp. 22-25 (2013)

7. Kumar, R.R., Mazanek, D.D., Heck, M.L.: Simulation and shuttle hitchhiker validation of passive satellite aerostabilization. J. Spacecr, Rockets (1995)

8. United Nations Office For Outer Space Affairs and Committee on the Peaceful Uses of Outer Space, "Space Debris Mitigation Guidelines of the Committee on the Peaceful Uses of Outer Space," (2010)

9. Yang, X.: Low earth orbit (LEO) mega constellations—satellite and terrestrial integrated communication networks. University of Surrey (2018)

10. Speretta, S., Sundaramoorthy, P., Gill, E.K.A.: Long-term performance analysis of NORAD two-line elements for CubeSats and PocketQubes. In: 11th IAA Symposium on Small Satellites for Earth Observation (2017)

11. California Polytechnic State University. Cubesat design specification. (2009)

12. ITU. Provisional final acts WRC-19 (2019)

13. Fonod, R., Gill, E.: Magnetic detumbling of fast-tumbling picosatellites. In: Proceedings of the International Astronautical Congress, IAC (2018)

14. Vergoossen, T., Guo, J., Bouwmeester, J., Groen, W.A.: Design, integration, and testing of world's smallest satellite reaction wheel. In: Proceedings of the International Astronautical Congress, IAC (2017)
15. Pallichadath, A., Radu, V., de Athayde Costa e Silva, S., Guerrieri, M., Cervone, D.: Integration and miniaturization challenges in the design of micro-propulsion systems for picosatellite platforms. In: Space Propulsion Conference (2018)

16. Crisp, N., Smith, K., Hollingsworth, P.: Small satellite launch to LEO: a review of current and future launch systems. Trans. Japan Soc. Aeronaut. Sp. Sci. Aerosp. Technol. Japan 12, 1-9 (2014)

17. Barnhart, D.J., Sweeting, M.N.: Right-sizing small satellites David. In: Proceedings of the AIAA/USU Conference on Small Satellites, pp. 1-8 (2014)

18. Bouwmeester, J., Gill, E., Speretta, S., Uludag, S.: New approach on the physical architecture of CubeSats \& PocketQubes. In: Proceedings of the 15th Reinventing Space Conference, pp. 1-13 (2017)

19. Boshuizen, C.R., Mason, J., Klupar, P., Spanhake, S.: Results from the planet labs flock constellation. In: 28th Annual AIAA/USU Conference on Small Satellites, pp. SSC14-I-1 (2014)

20. Selva, D., Krejci, D.: A survey and assessment of the capabilities of Cubesats for earth observation. Acta Astronaut. 74, 50-68 (2012)

21. Miles, D.M., et al.: A miniature, low-power scientific fluxgate magnetometer: a stepping-stone to cube-satellite constellation missions. J. Geophys. Res. Sp. Phys. (2016)

22. Olsen, N., Moretto, T., Friis-Christensen, E.: New approaches to explore the earth's magnetic field. J. Geodyn. 33(1-2), 29-41 (2002)

23. Mitchell, E.F., et al.: The highly miniaturised radiation monitor. J. Instrum. 9(7) (2014)

24. Shibasaki, D.M.R..: Presentation: low-cost GNSS receiver for high-precision GNSS data processing. In: COPUOS Meeting (2019)

25. Jäggi, A., Bock, H., Prange, L., Meyer, U., Beutler, G.: GPS-only gravity field recovery with GOCE, CHAMP, and GRACE. Adv. Sp. Res. 47(6), 1020-1028 (2011)

26. Cajacob, D., Mccarthy, N., O'Shea, T., McGwier, R.: Geolocation of RF emitters with a formation-flying cluster of three microsatellites. In: 30th Annual AIAA/USU Conference on Small Satellites (2016)

27. Dudas, L., Szucs, L., Gschwindt, A.: The spectrum monitoring system by Smog-1 satellite. In: Proceedings of 14th Conference on Microwave Techniques, COMITE 2015 (2015)

28. Birkeland, R., Hornig, A.: On how a CubeSat swarm can improve the coverage for an Arctic ground based sensor network. In: The 4S symposium, June, pp. 1-13 (2016)

29. Speretta S, et al.: LUMIO: an autonomous CubeSat for lunar exploration. In: Space Operations: Inspiring Humankind's Future (2019)

30. Staehle, R., Blaney, D., Hemmati, H.: Interplanetary CubeSats: opening the solar system to a broad community at lower cost. J. Small Satell. 2(1), 161-186 (2013)

31. Parkin, K. L. G.: The breakthrough starshot system model. Acta Astronaut. (2018)

Publisher's Note Springer Nature remains neutral with regard to jurisdictional claims in published maps and institutional affiliations. 